\title{
Correlations Between First Documented Cardiac Rhythms and Preceding Telemetry in Patients With Code Blue Events
}

Utpal S. Bhalala, MD¹, Christopher P. Bonafide, MD, MSCE², Christian M. Coletti, MD*, Penny E. Rathmanner, RN, BSN , Vinay M. Nadkarni, MD, MS ${ }^{5}$, Robert A. Berg, MD5, Anita K. Witzke, MSN, RN, Melody S. Kasprzak, PhD7, Marc T. Zubrow, MD

${ }^{1}$ Department of Anesthesia and Critical Care Medicine and Pediatrics, Johns Hopkins Hospital and Johns Hopkins University School of Medicine, Baltimore, Maryland; '2Department of Pediatrics, The Children's Hospital of Philadelphia and Perelman School of Medicine, University of Pennsylvania, Philadelphia, Pennsylvania; ${ }^{3}$ Department of Internal Medicine, Christiana Care Health System, Newark, Delaware; ${ }^{4}$ Department of Perioperative Services, Christiana Care Health System, Newark, Delaware; ${ }^{5}$ Department of Anesthesia and Critical Care Medicine and Pediatrics, The Children's Hospital of Philadelphia and Perelman School of Medicine, University of Pennsylvania, Philadelphia, Pennsylvania; ${ }^{6}$ Department of Information Technology, University of Maryland School of Medicine, Baltimore, Maryland; 'Department of Nursing, Christiana Care Health System, Newark, Delaware; ${ }^{8}$ Department of Medicine, University of Maryland School of Medicine, Baltimore, Maryland.

BACKGROUND: Among in-hospital cardiac arrest (IHCA) patients, the first cardiac rhythm documented on resuscitation records (FDR) is often used as a surrogate for arrest etiology. Although the FDR generally represents the electrical activity at the time of cardiopulmonary resuscitation initiation, it may not be the ideal rhythm to infer the arrest etiology. We hypothesized that a rhythm present earlier - at the time of the code blue call-would frequently differ from the FDR, because the FDR might represent the later stage of a progressive cardiopulmonary process.

OBJECTIVE: To evaluate agreement between FDR and telemetry rhythm at the time of code blue call.

DESIGN: Cross-sectional study.

SETTING: A 750-bed adult tertiary care hospital and a 240bed adult inner city community hospital.

PATIENTS: Adult general ward patients monitored on the hospital's telemetry system during the 2 minutes prior to a code blue call for IHCA.
INTERVENTION: None.

MEASUREMENTS: Agreement between FDR and telemetry rhythm.

RESULTS: Among 69 IHCAs, agreement between FDR and telemetry was $65 \%$ (kappa $=0.37$ ). Among 17 events with FDRs of ventricular tachyarrhythmia (VTA), telemetry showed VTA in 12 (71\%) and other organized rhythms in 5 (29\%). Among 12 events with first documented rhythms of asystole, telemetry showed asystole in $3(25 \%)$, VTA in 1 $(8 \%)$, and other organized rhythms in 8 (67\%).

CONCLUSIONS: The FDR had only fair agreement with the telemetry rhythm at the time of code blue call. The telemetry rhythm may be a useful adjunct to the FDR when investigating arrest etiology. Journal of Hospital Medicine 2013;8:225-228. (C) 2013 Society of Hospital Medicine.
In-hospital cardiac arrest (IHCA) research often relies on the first documented cardiac rhythm (FDR) on resuscitation records at the time of cardiopulmonary resuscitation (CPR) initiation as a surrogate for arrest etiology. ${ }^{1}$ Over 1000 hospitals report the FDR and associated cardiac arrest data to national registries annually. ${ }^{2,3}$ These data are subsequently used to report national IHCA epidemiology, as well as to develop and refine guidelines for in-hospital resuscitation. ${ }^{4}$

Suspecting that the FDR might represent the later stage of a progressive cardiopulmonary process rather than a sudden dysrhythmia, we sought to compare the

*Address for correspondence and reprint requests: Christian Coletti, MD, Doctors for Emergency Service and Internal Medicine Clinic, Christiana Care Health System, 4755 Ogletown-Stanton RD, Newark, DE 19718; Telephone: 302-733-1840; Fax: 302-733-1533; E-mail: ccoletti@christianacare.org

Additional Supporting Information may be found in the online version of this article.

Received: November 20, 2012; Revised: January 27, 2013; Accepted: February 1, 2013

2013 Society of Hospital Medicine DOI 10.1002/jhm.2028

Published online in Wiley Online Library (Wileyonlinelibrary.com). first rhythm documented on resuscitation records at the time of CPR initiation with the telemetry rhythm at the time of the code blue call. We hypothesized that the agreement between FDR and telemetry rhythm would be $<80 \%$ beyond that predicted by chance $($ kappa $<0.8) .{ }^{5}$

\section{METHODS \\ Design}

Between June 2008 and February 2010, we performed a cross-sectional study at a 750-bed adult tertiary care hospital (Christiana Hospital) and a 240-bed adult inner city community hospital (Wilmington Hospital). Both hospitals included teaching and nonteaching inpatient services. The Christiana Care Health System Institutional Review Board approved the study.

\section{Study Population}

Eligible subjects included a convenience sample of adult inpatients aged $\geq 18$ years who were monitored on the hospital's telemetry system during the 2 minutes prior to a code blue call from a nonintensive care, noncardiac care inpatient ward for IHCA. Intensive care 
TABLE 1. Resuscitation Record Rhythm Categorization Scheme

\begin{tabular}{ll}
\hline Category & Rhythm \\
\hline Asystole & Asystole \\
Ventricular tachyarrhythmia & Ventricular fibrillation, ventricular tachycardia \\
$\begin{array}{l}\text { Other organized } \\
\text { rhythms }\end{array}$ & Atrial fibrillation, bradycardia, paced pulseless \\
& electrical activity, sinus, idioventricular, other
\end{tabular}

unit (ICU) locations were excluded because they are not captured in our central telemetry recording system. We defined IHCA as a resuscitation event requiring $>1$ minute of chest compressions and/or defibrillation. We excluded patients with "do not attempt resuscitation" orders at the time of the IHCA. For patients with multiple IHCAs, only their first event was included in the analysis. International Classification of Diseases, 9th Revision admission diagnoses were categorized into infectious, oncology, endocrine/metabolic; cardiovascular, renal, or other disease categories. The decision to place patients on telemetry monitoring was not part of the study and was entirely at the discretion of the physicians caring for the patients.

\section{Variables and Measurements}

We reviewed the paper resuscitation records of each IHCA during the study period and identified the FDR. To create groups that would allow comparison between telemetry and resuscitation record rhythms, we placed each rhythm into 1 of the following 3 categories: asystole, ventricular tachyarrhythmia (VTA), or other organized rhythms (Table 1). It was not possible to retrospectively ascertain the presence of pulses to determine if an organized rhythm identified on telemetry tracings was pulseless electrical activity (PEA) or a perfusing rhythm. Therefore, we elected to take a conservative approach that would bias toward agreement (the opposite direction of our hypothesis that the rhythms are discrepant) and consider all other organized rhythms in agreement with one another. We reviewed printouts of telemetry electrocardiographic records for each patient. Minute 0 was defined as the time of the code blue call. Two physician investigators (C.C. and U.B.) independently reviewed telemetry data for each patient at minute 0 and the 2 minutes preceding the code blue call (minutes -1 and -2 ). Rhythms at each minute mark were assigned to 1 of the following categories according to the classification scheme in Table 1: asystole, VTA, or other organized rhythms. Leads off and uninterpretable telemetry were also noted. Discrepancies in rhythm categorization between reviewers were resolved by a third investigator (M.Z.) blinded to rhythm category assignment. We used the telemetry rhythm at minute 0 for analysis whenever possible. If the leads were off or the telemetry was uninterpretable at minute 0 , we used minute -1 . If minute -1 was also unusable, we used minute -2 . If

\begin{tabular}{|c|c|c|}
\hline & $\mathrm{n}$ & $\%$ \\
\hline \multicolumn{3}{|l|}{ Age, y } \\
\hline $30-39$ & 1 & 1.4 \\
\hline $40-49$ & 4 & 5.8 \\
\hline $50-59$ & 11 & 15.9 \\
\hline $60-69$ & 15 & 21.7 \\
\hline $70-79$ & 16 & 23.2 \\
\hline $80-89$ & 18 & 26.1 \\
\hline $90+$ & 4 & 5.8 \\
\hline \multicolumn{3}{|l|}{ Sex } \\
\hline Male & 26 & 37.7 \\
\hline Female & 43 & 62.3 \\
\hline \multicolumn{3}{|l|}{ Race/ethnicity } \\
\hline White & 51 & 73.9 \\
\hline Black & 17 & 24.6 \\
\hline Hispanic & 1 & 1.4 \\
\hline \multicolumn{3}{|l|}{ Admission body mass index } \\
\hline Underweight (<18.5) & 3 & 4.3 \\
\hline Normal $(18.5-<25)$ & 15 & 21.7 \\
\hline Overweight $(25-<30) 24$ & 24 & 34.8 \\
\hline Obese $(30-<35)$ & 17 & 24.6 \\
\hline Very obese $(\geq 35)$ & 9 & 13.0 \\
\hline Unknown & 1 & 1.4 \\
\hline \multicolumn{3}{|l|}{ Admission diagnosis category } \\
\hline Infectious & 29 & 42.0 \\
\hline Oncology & 4 & 5.8 \\
\hline Endocrine/metabolic & 22 & 31.9 \\
\hline Cardiovascular & 7 & 10.1 \\
\hline Renal & 2 & 2.8 \\
\hline Other & 5 & 7.2 \\
\hline
\end{tabular}

there were no usable data at minutes $0,-1$, or -2 , we excluded the patient.

\section{Statistical Analysis}

We determined the percent agreement between the resuscitation record rhythm category and the last interpretable telemetry rhythm category. We then calculated an unweighted kappa for the agreement between the resuscitation record rhythm category and the last interpretable telemetry rhythm category.

\section{RESULTS}

During the study period, there were 135 code blue calls for urgent assistance among telemetry-monitored non-ICU patients. Of the 135 calls, we excluded 4 events $(3 \%)$ that did not meet the definition of IHCA, 9 events $(7 \%)$ with missing or uninterpretable data, and 53 events $(39 \%)$ with unobtainable data due to automatic purging from the telemetry server. Therefore, 69 events in 69 different patients remained for analysis. Twelve of the 69 included arrests that occurred at Wilmington Hospital and 57 at Christiana Hospital. The characteristics of the patients are shown in Table 2.

Of the 69 arrests, we used the telemetry rhythm at minute 0 in 42 patients $(61 \%)$, minute -1 in 22 patients $(32 \%)$, and minute -2 in 5 patients $(7 \%)$. Agreement between telemetry and FDR was $65 \%$ $($ kappa $=0.37,95 \%$ confidence interval: $0.17-0.56)$ 
TABLE 3. Agreement Between Telemetry at Time of Code Call and First Documented Resuscitation Record Rhythm

\begin{tabular}{lllll}
\hline & \multicolumn{4}{c}{ Resuscitation Record } \\
\cline { 2 - 5 } Telemetry & Asystole & $\begin{array}{l}\text { Ventricular } \\
\text { Tachyarrhythmia }\end{array}$ & $\begin{array}{l}\text { Other } \\
\text { Organized } \\
\text { Rhythms }\end{array}$ & Total \\
\hline Asystole & 3 & 0 & 2 & 5 \\
Ventricular tachyarrhythmia & 1 & 12 & 8 & 21 \\
Other organized rhythms & 8 & 5 & 30 & 43 \\
Total & 12 & 17 & 40 & 69 \\
\hline
\end{tabular}

NOTE: Agreement between telemetry and resuscitation record is shown in bold.

(Table 3). Agreement did not vary significantly by sex, race, hospital, weekday, time of day, or minute used in the analysis. Agreement was not associated with survival to hospital discharge.

Of the 69 IHCA events, the FDRs vs telemetry rhythms at the time of IHCA were: asystole $17 \%$ vs $7 \%$, VTA $25 \%$ vs $31 \%$, and other organized rhythms $58 \%$ vs $62 \%$. Among the 12 events with FDR recorded as asystole, telemetry at the time of the code call was asystole in $3(25 \%)$, VTA in $1(8 \%)$, and other organized rhythms in $8(67 \%)$. Among the 17 events with FDR recorded as VTA, telemetry at the time of the code call was VTA in $12(71 \%)$ and other organized rhythms in $5(29 \%)$. Among the 40 events with FDR recorded as other organized rhythms, telemetry at the time of the code call was asystole in 2 $(5 \%)$, VTA in $8(20 \%)$, and other organized rhythms in $30(75 \%)$. Among the 8 patients with VTA on telemetry and other organized rhythms on the resuscitation record, the other organized rhythms were documented as PEA $(n=6)$, sinus $(n=1)$, and bradycardia $(\mathrm{n}=1)$. Of the 12 patients with VTA on telemetry and on the resuscitation record, 8 (67\%) had ventricular tachycardia on telemetry. Four of the $8(50 \%)$ who had ventricular tachycardia on telemetry had deteriorated into ventricular fibrillation by the time the FDR was recorded. Of the 4 who had ventricular fibrillation on telemetry, all had ventricular fibrillation as the FDR on the resuscitation record.

\section{DISCUSSION}

These results establish that FDRs often differ from the telemetry rhythms at the time of the code blue call. This is important because national registries such as the American Heart Association's Get with the Guidelines-Resuscitation ${ }^{2}$ database use the FDR as a surrogate for arrest etiology, and use their findings to report national IHCA outcomes as well as to develop and refine evidence-based guidelines for in-hospital resuscitation. Our findings suggest that using the FDR may be an oversimplification of the complex progression of cardiac rhythms that occurs in the periarrest period. Adding preceding telemetry rhythms to the data elements collected may shed additional light on etiology. Furthermore, our results demonstrate that, among adults with VTA or asystole documented upon arrival of the code blue team, other organized rhythms are often present at the time the staff recognized a lifethreatening condition and called for immediate assistance. This suggests that the VTA and asystole FDRs may represent the later stages of progressive cardiopulmonary processes. This is in contrast to out-ofhospital cardiac arrests typically attributed to sudden catastrophic dysrhythmias that often progress to asystole unless rapidly defibrillated. ${ }^{6-8}$ Out-of-hospital and in-hospital arrests are likely different (but overlapping) entities that might benefit from different resuscitation strategies. ${ }^{9,10}$ We hypothesize that, for a subset of these patients, progressive respiratory insufficiency and circulatory shock-conditions classically associated more strongly with pediatric than adult IHCA-may have been directly responsible for the event. ${ }^{1}$ If future research supports the concept that progressive respiratory insufficiency and circulatory shock are responsible for more adult IHCA than previously recognized, more robust monitoring may be indicated for a larger subset of adult patients hospitalized on general wards. This could include pulse oximetry (wave form can be a surrogate for perfusion), respiratory rate, and/or endtidal $\mathrm{CO}_{2}$ monitoring. In addition, if future research confirms that there is a greater distinction between inhospital and out-of-hospital cardiac arrest etiology, the expert panels that develop resuscitation guidelines should consider including setting of resuscitation as a branch point in future algorithms.

Our study had several limitations. First, the sample size was small due to uninterpretable rhythm strips, and for $39 \%$ of the total code events, the telemetry data had already been purged from the system by the time research staff attempted to retrieve it. Although we do not believe that there was any systematic bias to the data analyzed, the possibility cannot be completely excluded. Second, we were constrained by the inability to retrospectively ascertain the presence of pulses to determine if an organized rhythm identified on telemetry tracings was PEA. Thus, we categorized rhythms into large groups. Although this limited the granularity of the rhythm groups, it was a conservative approach that likely biased toward agreement (the opposite direction of our hypothesis). Third, the lack of perfect time synchronization between the telemetry system, wall clocks in the hospital, and wrist watches that may be referenced when documenting resuscitative efforts on the resuscitation record means that the rhythms we used may have reflected physiology after interventions had already commenced. Thus, in some situations, minute $-1,-2$, or earlier minutes may more accurately reflect the preintervention rhythm. Highly accurate time synchronization should be a central component of future prospective work in this area. 


\section{CONCLUSIONS}

The FDR had only fair agreement with the telemetry rhythm at the time of the code blue call. Among those with VTA or asystole documented on CPR initiation, telemetry often revealed other organized rhythms present at the time hospital staff recognized a life-threatening condition. In contrast to out-of-hospital cardiac arrest, FDR of asystole was only rarely preceded by VTA, and FDR of VTA was often preceded by an organized rhythm. ${ }^{8,11}$ Future studies should examine antecedent rhythms in combination with respiratory and perfusion status to more precisely determine arrest etiology.

\section{Acknowledgments}

The authors thank the staff at Flex Monitoring at Christiana Care Health System for their vital contributions to the study.

\section{Disclosures}

Dr. Zubrow had full access to all the data in the study and takes responsibility for the integrity of the data and the accuracy of the data analysis. The authors report no conflicts of interest.

\section{References}

1. Nadkarni VM, Larkin GL, Peberdy MA, et al. First documented rhythm and clinical outcome from in-hospital cardiac arrest among children and adults. JAMA. 2006;295(1):50-57.
2. Get With The Guidelines-Resuscitation (GWTG-R) overview. Available at: http://www.heart.org/HEARTORG/Healthcare Research/GetWithTheGuidelines-Resuscitation/Get-With-The-Guide lines-ResuscitationOverview_UCM_314497_Article.jsp. Accessed May $8,2012$.

3. Cummins RO, Chamberlain D, Hazinski MF, et al. Recommended guidelines for reviewing, reporting, and conducting research on inhospital resuscitation: the in-hospital "Utstein Style". Circulation. 1997;95:2213-2239.

4. Peberdy MA, Kaye W, Ornato JP, et al. Cardiopulmonary resuscitation of adults in the hospital: a report of 14,720 cardiac arrests from the National Registry of Cardiopulmonary Resuscitation. Resuscitation. 2003;58:297-308.

5. Landis JR, Koch GG. The measurement of observer agreement for categorical data. Biometrics. 1977;33(1):159-174.

6. Herlitz J, Bang A, Aune S, et al. Characteristics and outcome among patients suffering in-hospital cardiac arrest in monitored and nonmonitored areas. Resuscitation. 2001;48:125-135.

7. Herlitz J, Bang A, Ekstrom L, et al. A comparison between patients suffering in-hospital and out-of hospital cardiac arrest in terms of treatment and outcome. I Intern Med. 2000;248:53-60.

8. Fredriksson M, Aune S, Bang A, et al. Cardiac arrest outside and inside hospital in a community: mechanisms behind the differences in outcomes and outcome in relation to time of arrest. Am Heart J. 2010;159:749-756.

9. Weisfeldt ML, Everson-Stewart S, Sitlani C, et al.; Resuscitation Outcomes Consortium Investigators. Ventricular tachyarrhythmias after cardiac arrest in public versus at home. N Engl J Med. 2011;364: 313-321.

10. Monteleone PP, Lin CM. In-hospital cardiac arrest. Emerg Med Clin North Am. 2012;30:25-34.

11. Holmgren C, Bergfeldt L, Edvardsson N, et al. Analysis of initial rhythm, witnessed status and delay to treatment among survivors of out-of-hospital cardiac arrest in Sweden. Heart. 2010;96: 1826-1830. 